\title{
Analyzing X-ray pulsar profiles: geometry and beam pattern of EXO 2030+375
}

\author{
M. Sasaki ${ }^{1}$, D. Klochkov ${ }^{1}$, U. Kraus ${ }^{2}$, I. Caballero ${ }^{3}$, and A. Santangelo ${ }^{1}$ \\ ${ }^{1}$ Institut für Astronomie und Astrophysik, Universität Tübingen, Sand 1, 72076 Tübingen, Germany \\ e-mail: sasaki@astro.uni-tuebingen.de \\ 2 Institut für Physik und Technik, Universität Hildesheim, Marienburger Platz 22, 31141 Hildesheim, Germany \\ 3 CEA Saclay, DSM/IRFU/SAp - UMR AIM (7158) CNRS/CEA/Université P. Diderot, 91191 Gif-sur-Yvette, France
}

Received 14 December 2009 / Accepted 7 April 2010

\section{ABSTRACT}

\begin{abstract}
Context. The pulse profiles of the transient Be/X-ray binary EXO 2030+375 show strong dependence on energy, as well as on its luminosity state, and are asymmetric in shape.

Aims. We want to identify the emission components of the two magnetic poles in the pulsed emission to understand the geometry of the neutron star and its beam pattern.

Methods. We utilize a pulse-profile decomposition method that enables us to find two symmetric pulse profiles from the magnetic poles of the neutron star. The symmetry characteristics of these single-pole pulse profiles give information about the position of the magnetic poles of the neutron star relative to its rotation axis.

Results. We find a possible geometry for the neutron star in EXO 2030+375 through the decomposition of the pulse profiles, which suggests that one pole gets closer to the line of sight than the other and that, during the revolution of the neutron star, both poles disappear behind the horizon for a short period of time. A considerable fraction of the emission arises from a halo while the pole is facing the observer and from the accretion stream of the other pole while it is behind the neutron star, but the gravitational line bending makes the emission visible to us.
\end{abstract}

Key words. stars: neutron - X-rays: binaries

\section{Introduction}

EXO $2030+375$ is an accreting X-ray pulsar with a pulsation period of $\sim 42 \mathrm{~s}$, which was discovered with EXOSAT in 1985 during a giant outburst (Parmar et al. 1989b). A B0 Ve star was found as its counterpart in follow-up observations in the optical and infrared bands (Janot-Pacheco et al. 1988; Motch \& Janot-Pacheco 1987; Coe et al. 1988). During the giant outburst, EXO $2030+375$ showed a spin-up of $-P / \dot{P} \approx 30$ yr (Parmar et al. 1989b) and quasi-periodic oscillations with a frequency of $\sim 0.2 \mathrm{~Hz}$ (Angelini et al. 1989) interpreted as caused by the formation of an accretion disk. Detailed analyses have shown that its rate of pulse-period change $\dot{P}$, energy spectrum, and pulse profile are strongly luminosity dependent (Parmar et al. 1989a,b; Reynolds et al. 1993). The orbital period is 46 days (Wilson et al. 2002), and a normal outburst has been detected for nearly every periastron passage since 1991 (Wilson et al. 2005). In 2006, EXO 2030+375 underwent the first giant outburst since its discovery in 1985 (Corbet \& Levine 2006; Krimm et al. 2006; McCollough et al. 2006), during which it reached a maximum luminosity of $L_{1-20 \mathrm{keV}} \approx 1.2 \times 10^{38} \mathrm{erg} \mathrm{s}^{-1}$ (Klochkov et al. 2008) and again showed a strong spin-up. Rossi X-ray Timing Explorer ( RXTE) monitored EXO 2030+375 extensively during the 2006 giant outburst (Wilson et al. 2008). The source was also observed by the INTErnational Gamma Ray Astrophysics Laboratory (INTEGRAL, Winkler et al. 2003) and Swift (Gehrels et al. 2004). The spectra indicate a cyclotron absorption line (Klochkov et al. 2007; Wilson et al. 2008). Klochkov et al. (2008) have shown that the spectrum of EXO 2030+375 changes with pulse phase, suggesting a fan beam geometry during the maximum, while towards the end of the giant outburst, it changes to a combination of a fan beam and a pencil beam.

In X-ray pulsars, a neutron star accretes matter from a companion star via stellar wind or Roche lobe overflow. The accreted matter is channeled along the field lines of the strong magnetic field of the neutron star onto the magnetic poles. X-ray emission from the neutron star is produced in regions around the two magnetic poles. As the magnetic dipole axis is most likely inclined against the rotation axis of the neutron star, a distant observer sees pulsed emission. X-ray pulsars exhibit a wide variety of pulse shapes that differ from source to source. Generally, high-energy pulses have simpler shapes than low-energy pulses (White et al. 1983; Frontera \& Dalfiume 1989; Bildsten et al. 1997, and references therein). If one assumes an axially symmetric geometry for the two emission regions of the neutron star in a dipole configuration, the observed pulse profile should be symmetric. However, the observed pulse profiles typically show an asymmetry. To explain the asymmetric shape of the total pulse profile, a distorted magnetic dipole field in which the two magnetic poles are not located opposite each other have been discussed (Parmar et al. 1989a; Leahy 1991; Riffert et al. 1993; Bulik et al. 1995). Kraus et al. (1995) shows that, starting from the observed, asymmetric pulse profile, it is possible to disentangle the contribution of the two emission regions of the neutron star. Once the pulsed emission from each of the poles has been obtained, one can derive the geometry of the neutron star. This again allows us to construct the beam pattern, i.e., the flux distribution from one emission region. Using this pulse-profile decomposition method, Kraus et al. (1996) have analyzed the 
pulse profiles of Cen $\mathrm{X}-3$ and find indications of both pencil and fan beam. In the case of Her X-1, the results of the pulseprofile decomposition by Blum \& Kraus (2000) have not only shed light on the beam pattern of the magnetic poles, but have also confirmed that a warped and tilted accretion disk attenuates the emission from one pole of the neutron star. For A $0535+26$, the reconstructed beam pattern suggests that the emission comes from a hollow column plus a halo of scattered radiation on the neutron star surface (Caballero et al. 2010).

In this paper we present the analysis of the energy-resolved pulse profiles of EXO 2030+375 utilizing the decomposition method developed by Kraus et al. (1995). Section 2 gives an overview of the data used for our analysis and Sect. 3 describes the analysis and the results obtained with the pulse-profile decomposition method. The results are discussed in Sect. 4. Section 5 summarizes the possible geometry of the neutron star and the origin of the observed emission.

\section{Data}

EXO 2030+375 experienced a giant outburst in 2006, during which the source was monitored continuously by $R X T E$ and was also observed by INTEGRAL. We have used the pulse profiles obtained with the Joint European X-Ray Monitor (JEM-X, Lund et al. 2003) and the imaging system IBIS/ISGRI (Ubertini et al. 2003) as presented in Figs. 2 and 8 of Klochkov et al. (2008).

\subsection{RXTE observations}

For better statistics, we also used publicly available archival data from two observations with RXTE during the rise and the decay of the giant outburst when EXO 2030+375 showed about half of the maximum luminosity. The observations took place on June 28 and September 17, 2006 (observation IDs 91089-0107-00 and 91089-01-19-01) when the luminosity of the source was $L_{1-20 \mathrm{keV}}=7 \times 10^{37} \mathrm{erg} \mathrm{s}^{-1}$ and $6 \times 10^{37} \mathrm{erg} \mathrm{s}^{-1}$, respectively. We started from the event files obtained with the Proportional Counter Array (PCA, Jahoda et al. 1996). We used the event encoded mode files in oder to have optimum binning. After filtering good time intervals and applying bitmasks, we created lightcurves with a time binning of $0.125 \mathrm{~s}$ in the following spectral bands: 2-9 keV, 9-14 keV, 14-20 keV, 20-30 keV, and $30-115 \mathrm{keV}$. After background subtraction, the lightcurves were corrected to solar barycenter and for orbitary motion of the binary. After folding the lightcurves with periods measured for each observation, we obtained pulse profiles with 128 phase bins. Phase 0.0 was fixed to agree with the INTEGRAL pulse profiles of Klochkov et al. (2008). The folded lightcurves from the RXTE observations are shown in Fig. 1.

During the two RXTE observations shown here, the luminosity of EXO 2030+375 was comparable. As can be seen in Fig. 1, the pulse profiles before the maximum and after the maximum of the giant outburst are very similar, corroborating that the shape of the pulse profiles only depends on the luminosity state (Parmar et al. 1989a).

\section{The analysis}

\subsection{The method}

A detailed description of the pulse-profile decomposition method, which is based on a backward tracing of the emission, can be found in Kraus et al. (1995). All major steps of our
Table 1. Parameters obtained from the decomposition for solutions 1 and 2.

\begin{tabular}{cccccccc}
\hline \hline & $\Phi_{1}$ & $\Delta$ & $a$ & $b$ & $\Theta_{1}{ }^{1}$ & $\Theta_{2}{ }^{1}$ & $\delta^{1}$ \\
\hline 1 & $65^{\circ}-75^{\circ}$ & $63^{\circ}-70^{\circ}$ & -2.1 & 1.0 & $39^{\circ}$ & $141^{\circ}$ & $40^{\circ}$ \\
2 & $70^{\circ}-80^{\circ}$ & $81^{\circ}-88^{\circ}$ & -0.1 & 1.0 & $87^{\circ}$ & $93^{\circ}$ & $85^{\circ}$ \\
\hline
\end{tabular}

(1) Assuming $\Theta_{0}=50^{\circ}$.

analysis and the criteria applied to obtain the best solution are described in the Appendix.

The basic assumption of the method is that the magnetic dipole field of the neutron star is distorted in such a way that the two magnetic poles do not lie on a straight line through the center of the neutron star. Therefore, even though the emission from each pole is axisymmetric, the sum of the emission from both poles results in an asymmetric pulse profile. Using Fourier analysis, we model the observed asymmetric pulse profiles with two symmetric functions $f_{1,2}$ to search for symmetry points $\Phi_{1,2}$ in the pulse profiles and their offset $\Delta$ (see Appendix for details). For each observation and energy range, the functions $f_{1}$ and $f_{2}$ correspond to the two single-pole pulse profiles that in total add up to the observed asymmetric pulse profile. Each symmetry point corresponds to the pulse phase where the respective pole is either closest to or most distant from the observer's line of sight. From the two symmetry points and functions, we then derive the location of the emission regions and the beam pattern.

\subsection{Decompositions}

We have a total of 26 pulse profiles from INTEGRAL and RXTE observations. To perform pulse-profile decomposition, the maximum in each of the 26 pulse profiles is normalized to unity. Thereafter each pulse profile is modeled with two symmetric functions $f_{1}$ and $f_{2}$ based on Fourier analysis. After applying criteria 1 (positive flux, see Appendix A.1) and 2 (no ripples), we obtain a large number of possible values for the parameters $\Phi_{1}$ and $\Delta$. However, after combining the results for all pulse profiles (criterion 3), only two interesting solution regions remain in the parameter space of $\Phi_{1}-\Delta$. We call these solutions 1 and 2 and perform further analysis with these two possible solutions.

For each observation and energy band, the functions $f_{1}$ and $f_{2}$ correspond to the two single-pole pulse profiles that in total add up to the observed asymmetric pulse profile. The Fourier analysis finds more than one possible set of $\Phi_{1}$ and $\Delta$ within a small region for one solution. For each total pulse profile, we have to look at the different sets of the single-pole pulse profiles to decide which one is consistent with the single-pole pulse profiles at other energies. The pulse profiles of the different observations are studied separately, because one should see a correlation between the different energy bands of one luminosity state, but not necessarily between two different observations. Figure 2 shows some of the selected single-pole pulse profiles and the derived beam patterns for each pole for solution 1 . Within one observation, one can see an energy-dependent evolution of the single-pole pulse profiles. The values of $\Phi_{1}$ and $\Delta$ for solutions 1 and 2 are $\left(65^{\circ}<\Phi_{1}<75^{\circ}, 63^{\circ}<\Delta<70^{\circ}\right)$ and $\left(70^{\circ}<\Phi_{1}<\right.$ $80^{\circ}, 81^{\circ}<\Delta<88^{\circ}$ ), respectively. The parameters for the two solutions are listed in Table 1. 
M. Sasaki et al.: Pulse profiles of EXO 2030+375
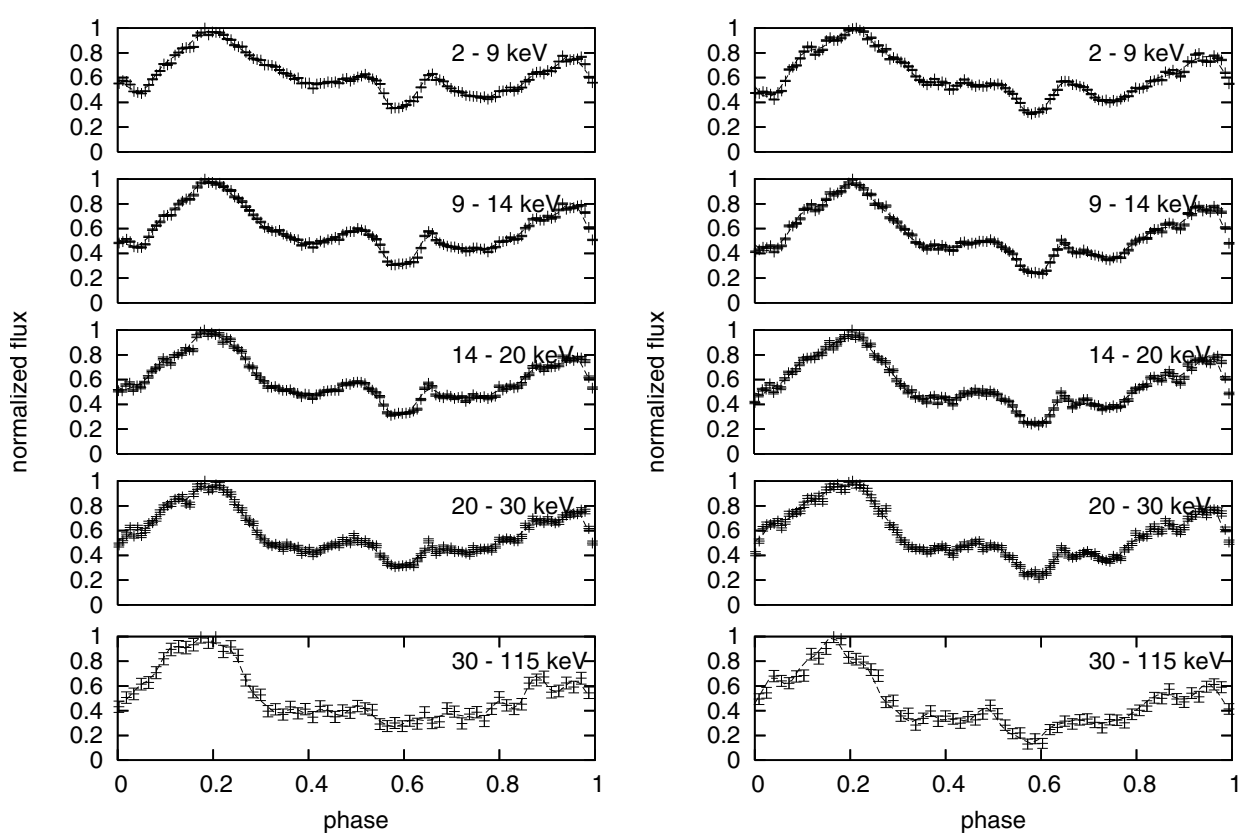

Fig. 1. Pulse profiles of EXO $2030+375$ during the rise (left) and the decay (right) of the giant outburst in 2006 observed with RXTE.

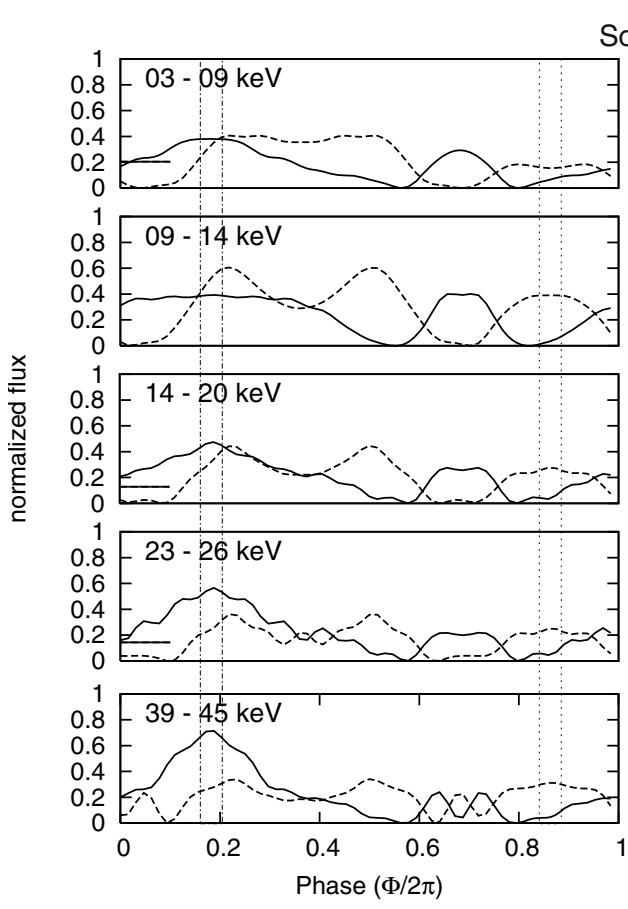

Solution 1

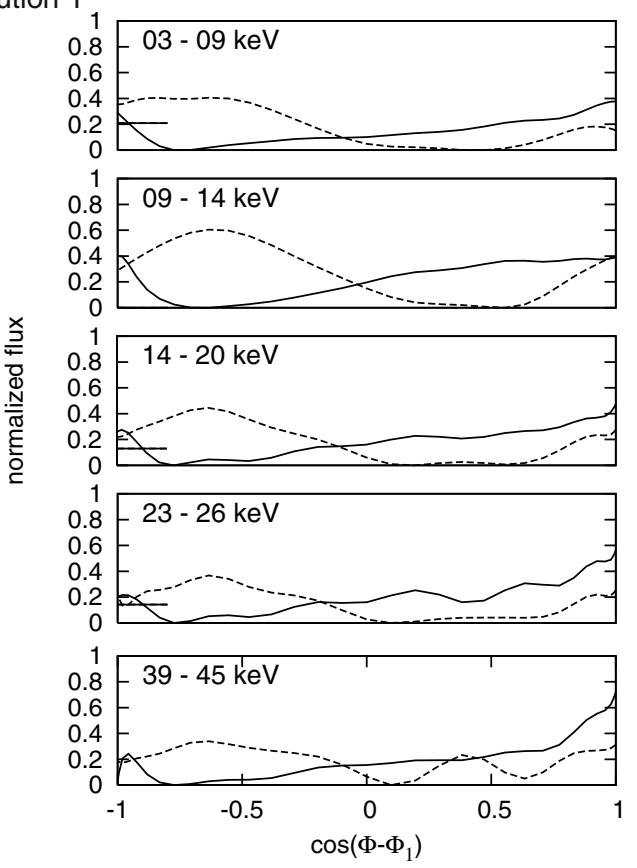

Fig. 2. A selection of single-pole pulse profiles (left) and corresponding beam patterns (right) obtained by the decomposition method for the $\Phi_{1}-\Delta$ set for solution 1 for the energy bands 3-9 keV, 9-14 keV, 14-20 keV, 23-26 keV, and $39-45 \mathrm{keV}$ of the INTEGRAL observation near maximum. The solid line is used for emission from the first pole, dashed line for the second pole. The vertical dash-dotted lines indicate the ranges for the symmetry point $\Phi_{1}$, while the dotted lines show the ranges for $\Phi_{2}$ obtained from pulse profiles of different energies and observations.

\subsection{Overlaying beam patterns}

As the neutron star rotates, the angle between the axis through one magnetic pole and the line of sight $\theta$ changes with phase, i.e., with rotation angle $\Phi$. The decomposition has provided us with beam patterns as seen by the distant observer for each emission region as functions of the phase. Now we compare the two beam patterns derived from the two single-pole pulse profiles of each observed pulse profile and search for a range in $\cos \left(\Phi-\Phi_{1}\right)$, and thus $\theta$, in which the two beam patterns seem to show the same emission (see Appendix A.2). We try to overlay the two beam patterns by using the relation:

$\cos \left(\Phi-\Phi_{1}\right)=a+b \cos \left(\tilde{\Phi}-\Phi_{2}\right), \quad b>0$.

For solution 1 we are not able to find an overlap of the singlepole beam patterns. It is more likely that, in this case, the geometry only allows the observer to see two different parts of the total beam pattern. To assemble the two parts of the beam pattern, we use $a=-2.1$ and $b=1.0$ (see Fig. A.2, upper panel). For solution 2, we find an overlap of the single-pole beam patterns for $a=-0.1, b=1.0$, although the beam patterns do not seem to match perfectly.

A total beam pattern can be reconstructed from the beam patterns calculated from the single-pole pulse profiles. As shown in Kraus et al. (1995), there is an ambiguity in the relation between $\Phi$ and $\theta$, as each single-pole pulse profile has two symmetry points at $\Phi_{i}$ and $\Phi_{i}+\pi$. Therefore, the solutions cannot tell us which ends of the sections of the beam patterns belong to, e.g., the lower values of $\theta$. For each set of $\Phi_{1}$ and $\Delta$ we obtain two possible solutions (called plus and minus) for the total beam pattern. The decomposition method cannot tell us which one is the real solution. We have to take results from other measurements of the source into consideration, e.g., luminosities or 
Solution 1
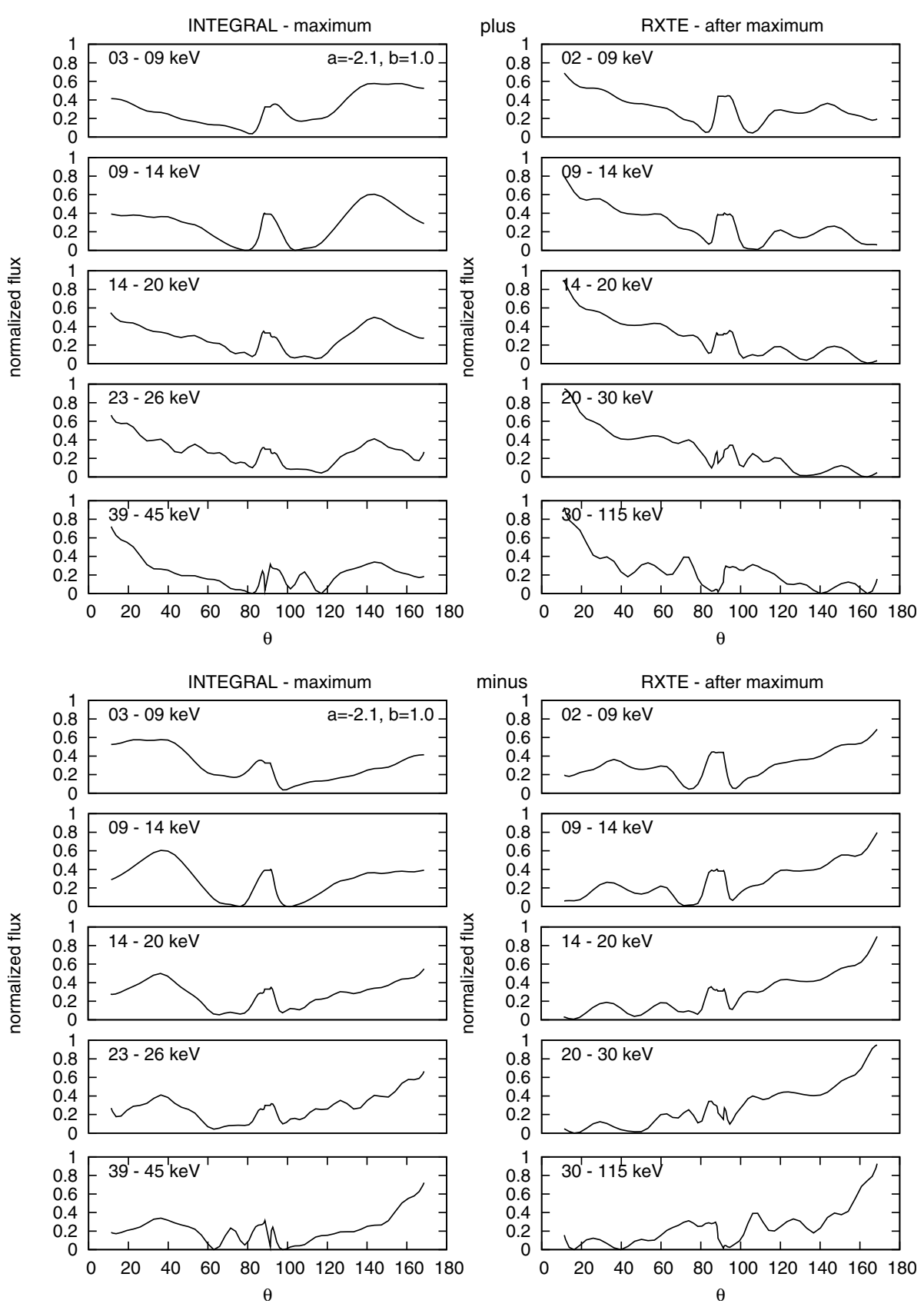

Fig. 3. Asymptotic beam patterns of the plus and minus solutions of solution 1 seen by the distant observer plotted over the angle $\theta$ between the first magnetic pole and the line of sight. The left panel shows the beam patterns of solution 1 of the observation near maximum as shown in Fig. 2. The right panel shows a selection of additional data from the observation after maximum with similar energy bands. spectra that will give insight into the emission processes, to decide which of the two is the real solution. The reconstructed asymptotic beam patterns for solution 1 as seen by the distant observer are shown in Fig. 3. Here the beam patterns are plotted over the angle $\theta$ between the normal at the first magnetic pole and the line of sight.

That the beam patterns of the single-pole pulse profiles do not match in the overlap region of solution 2 might indicate that the emission from the two magnetic poles are not identical and cannot be described with one local beam pattern. Therefore, we take the mean of the two beam patterns and model a total averaged beam pattern, which we use to reconstruct the visible total pulse profile, i.e., assuming equal local emission pattern for the two poles. The reconstructed pulse profiles (Fig. A.3) show significant deviations from the observed profiles. In addition, this solution yields a very extreme geometry with the two magnetic poles located near the equator of the neutron star, forming an angle of $\sim 90^{\circ}$ between each other (see Table 1). Although, in principle, we cannot rule such a strongly distorted geometry out, especially not for young neutron stars like those expected in Be/X-ray binary systems, as they might have experienced some anisotropic conditions while their birth, this rather unlikely geometry also suggests that solution 2 is not appropriate for EXO $2030+375$. Therefore, in the following, we focus on the discussion of solution 1.

\subsection{Geometry of the neutron star}

To derive the exact geometry of the neutron star, i.e., to determine the polar angles of the magnetic poles $\Theta_{1}$ and $\Theta_{2}$ as well as the offset angle $\delta$, we need to know the inclination angle of the rotation axis of the neutron star. However, this angle 


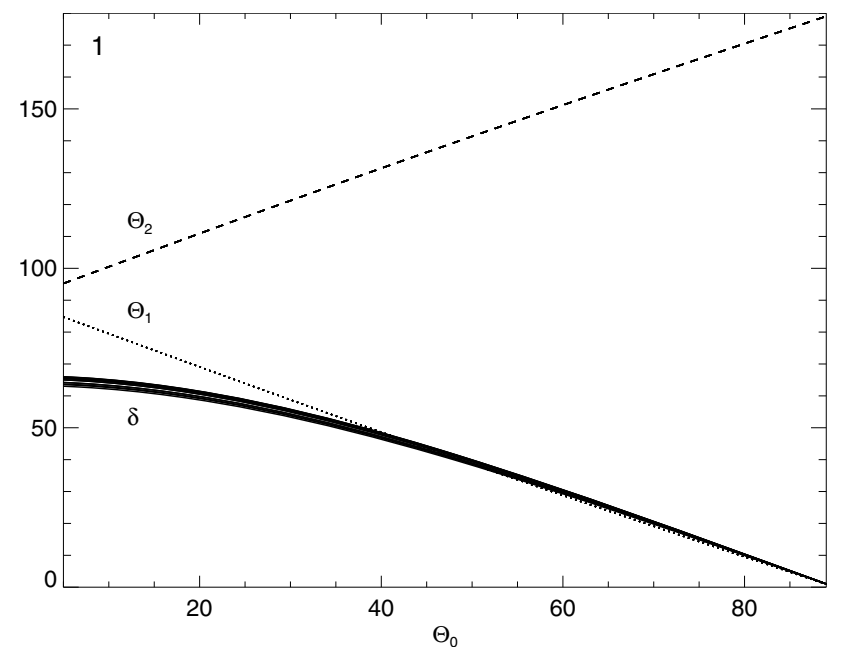

Fig. 4. Relation between the observing angle $\Theta_{0}$ and the angles $\Theta_{1}$ (dotted line), $\Theta_{2}$ (dashed line), and $\delta$ (range of possible values) for solution 1.

is not known for most of the neutron stars, in particular not for EXO 2030+375. Figure 4 shows the dependence of the angles $\Theta_{1}, \Theta_{2}$, and $\delta$ on the angle $\Theta_{0}$ between the rotation axis of the neutron star and the line of sight of the observer. To convert the phase parameter $\cos \left(\Phi-\Phi_{1}\right)$ into the angle $\theta$ between the first magnetic pole and the observer, we have to assume an inclination angle $\Theta_{0}$ with respect to the rotation axis. Here, we use $\Theta_{0}=50^{\circ}$, corresponding to $\Theta_{1}=39^{\circ}, \Theta_{2}=141^{\circ}$ (see Fig. 4 and Table 1).

\section{Discussion}

In this section we want to further examine the results of the decomposition method and present the possible geometry of the neutron star.

\subsection{Disentangling the emission components}

For solution 1, there is no overlap between the two beam patterns obtained from the single-pole pulse profiles as shown in Sect. 3.3. It means that only a part of the emission is seen from each pole during the revolution of the neutron star. By putting the two visible parts together, we obtain the total beam pattern of the emission around one magnetic pole. In doing so, we make the assumption that the two magnetic poles have the same emission pattern. The plus solution (Fig. 3, upper panel) can be described as a composition of a forward directed emission (towards $0^{\circ}$ ) that is more pronounced at higher energies and an extended, relatively soft emission $\left(\sim 80^{\circ}-180^{\circ}\right)$. The harder, forward-directed emission indicates a pencil beam. The relative flux of the softer emission component is higher in the data from the observation performed near the maximum compared to the one during the decay and can be interpreted as a fan beam, in agreement with the largely accepted picture that an optically thick accretion column is formed during the giant outburst (White et al. 1983, and references therein). The minus solution has a soft emission at $\theta \lesssim$ $60^{\circ}$ and a harder emission at $\theta \gtrsim 120^{\circ}$ that increases for larger $\theta$. As newest calculations by Kraus et al. (2010) have shown, reprocessing of photons in the upper accretion stream creates a significant emission component that dominates the beam pattern at higher energies and can be observed while the pole, hence the accretion column, is on the other side of the neutron star ("anti-pencil"). In addition, the emission from a halo that is

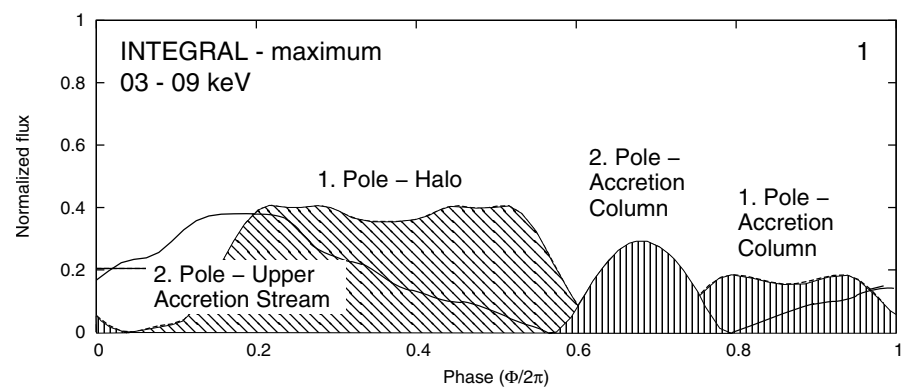

Fig. 5. Decomposed single-pole pulse profiles of the data taken near the maximum of the giant outburst for the lowest energy band (same as the upper left diagram of the upper [solution 1] panels in Fig. 2) with proposed emission components derived from the beam patterns of the minus solution (Fig. 3, lower panel). We suggest that the emission from the first pole consists of emission from the halo (diagonally striped component) and from the accretion column (vertically striped). The dominant emission from the second pole seems to have its origin in the scattered photons in the upper accretion stream, which is visible while the second pole is behind the horizon of the neutron star due to gravitational light bending, while there is also a contribution from the accretion column (vertically striped). In this solution, the first pole gets closer to the line of sight than the second.

formed by scattered photons at the bottom of the accretion column dominates the beam pattern at lower energies and at lower $\theta$ and is stronger in the data near the maximum of the giant outburst.

Figure 5 shows how the emission from the two poles contributes to the pulse profiles for the decompositions of the low energy band data taken at the maximum of the giant outburst in the case of the minus solution. Let us assume that the first magnetic pole with the polar angle $\Theta_{1}$ gets closer to the line of sight than the second magnetic pole, i.e., $\Theta_{0}-\Theta_{1} \leq \Theta_{2}-\Theta_{0}$. At phase 0.0 , both poles are right behind the horizon of the neutron star: the first pole is going to reappear, the second pole is turning farther away from the observer. At phase $\sim 0.1$ the first pole becomes visible, and from then on the emission from the halo of the first pole makes the largest contribution until the pole disappears behind the horizon at $\sim 0.6$. When the first pole, which is closer to the observer's line of sight, is right at the horizon of the neutron star and its accretion column is seen from the side, a minimum is likely to be observed in the pulse profile (see also Sect.4.4). At the major maximum at phase $0.15-0.25$, the second magnetic pole is behind the horizon of the neutron star and the scattered and gravitationally bent photons from the upper accretion stream cause the pronounced increase in flux. The second pole that is rotating on a circle farther away from the line of sight than the first pole comes back to the front side at about phase 0.45 and is closest to the observer at about phase 0.7. However, since the line of sight is closer in latitude to the first pole than to the second pole, the observer never gets as close to the surface normal of the second pole as to that of the first pole. The main emission seen from the second pole while it is on this side of the horizon comes directly from the accretion column.

Parmar et al. (1989a) have modeled the luminosity dependent pulse profiles from the first observed giant outburst in 1985 by assuming a fan beam and a pencil beam component for the two magnetic poles based on a model by Wang \& Welter (1981). They obtain a fit for all ten pulse profiles for different luminosities ranging from $0.1-10.0 \times 10^{37} \mathrm{erg} \mathrm{s}^{-1}$ with some residuals and find that the fan beam mainly produces the major peak at phase 0.2 , whereas the peak at phase 0.95 can be ascribed to a pencil beam. The way they have chosen phase 0.0 is different 

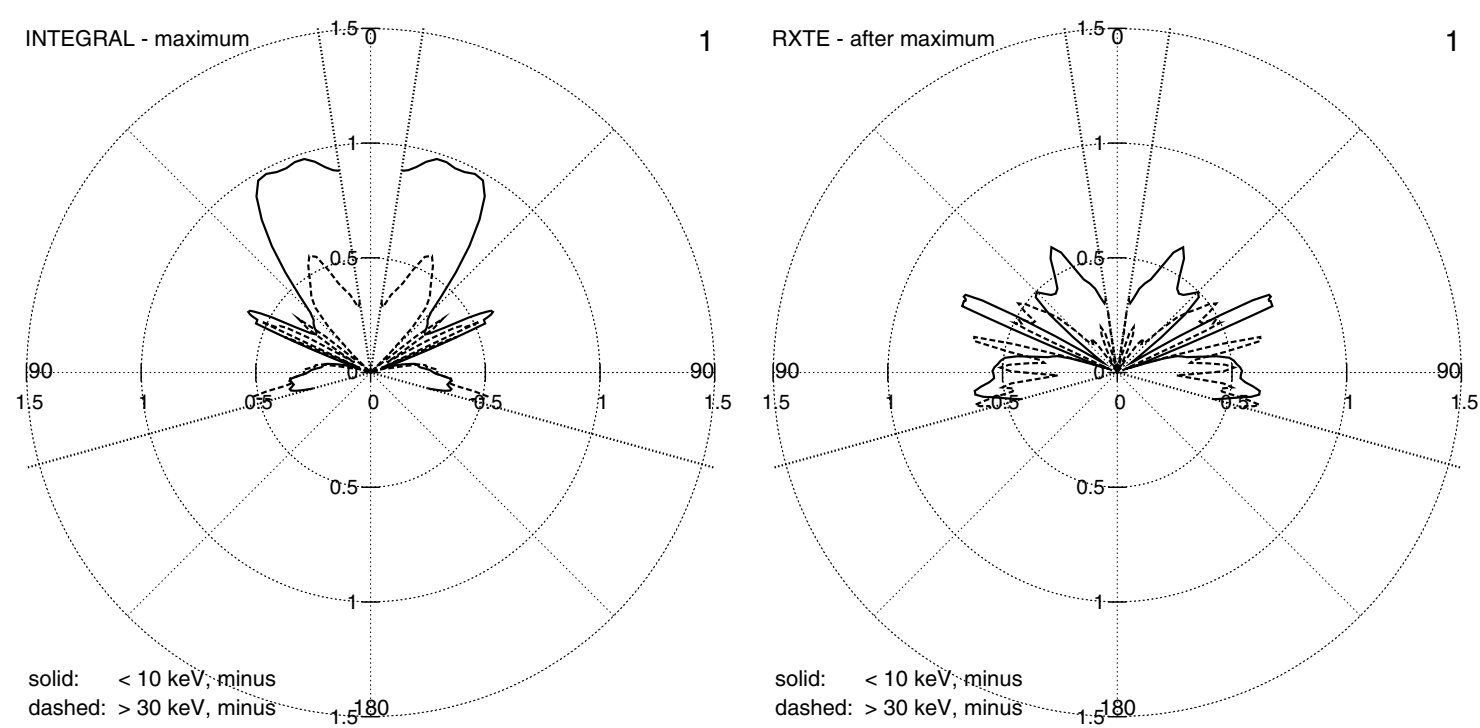

Fig. 6. Polar diagrams of the intrinsic beam pattern for the minus solution of solution 1 for the same observations as shown in Fig. 3, for the softest and the hardest bands for an assumed $\Theta_{0}=50^{\circ}$. Dotted lines are drawn to clearly show the range in which the emission is visible for the observer.

than in our work, resulting in a shift by about 0.4 . The best-fit parameters of Parmar et al. (1989a) correspond to $\Theta_{1}=70^{\circ}$, $\Theta_{2}=110^{\circ}$, and $\delta=70^{\circ}$, assuming $\Theta_{0}=25^{\circ}$. This result is consistent with our solution 1 for the case of $\Theta_{0}=25^{\circ}$ (see Fig. 4).

\subsection{Comparison to phase-resolved spectral analysis}

Klochkov et al. (2008) analyzed the same INTEGRAL data as used in this work and performed pulse phase resolved spectroscopy. For the data of the maximum, they find that the spectrum is harder at the main peak (phase 0.2). They conclude that the observer might be seeing Compton scattered photons from the optically thick accretion column. In the data taken at the end of the decay, a new peak appears at about phase 0.95. At this point, the spectrum is again harder, leading to the conclusion that the line of sight of the observer is closest to the magnetic field lines. This picture is in good agreement with the solution 1 of the decomposition, in which the emission of the upper accretion stream of the second pole, which is visible owing to gravitational light bending, has its maximum at phase $\sim 0.2$ while the halo of the first pole also has substantial emission, thus contributing significantly to the main peak. At phase $\sim 0.95$ the scattered emission from the upper accretion stream of the second pole starts to increase, while the emission from the accretion column of the first pole also contributes to the observed emission.

\subsection{Intrinsic beam patterns}

The observed asymptotic beam pattern differs from the intrinsic local beam pattern of the emission region because of relativistic light deflection near the neutron star. Making assumptions on the radius $r$ and mass $m$ of the neutron star, the asymptotic angle $\theta$ can be transformed into the intrinsic angle $\vartheta$ relative to the surface normal at the first magnetic pole to describe the local emission pattern. We use the canonical values $r=10 \mathrm{~km}$ and $m=1.4 M_{\odot}$, thus the ratio between $r$ and the Schwarzschild radius $r_{\mathrm{S}}$ is $r / r_{\mathrm{S}}=2.4$. The intrinsic beam patterns of the minus solution are shown in Fig. 6 in polar diagrams. As can be seen in these polar diagrams, the range for the visible angle is $\sim 10^{\circ}-$ $105^{\circ}$ if $\Theta_{0}=50^{\circ}$ is assumed.

\subsection{Possible origin of the emission}

In X-ray pulsars, a strong magnetic field funnels matter accreted from the companion star onto the polar caps. The infalling particles deposit their energy in the atmosphere of the neutron star. Heating due to accretion is balanced by radiative cooling through bremsstrahlung emission, Compton scattering, and the so-called cyclotron radiation after collisional excitation of electrons. In low-luminosity states, the accreted matter is decelerated in the atmosphere of the neutron star by Coulomb scattering. Emission can be seen as pencil beams from the hot spots at polar caps (Wang \& Welter 1981). In high-luminosity states, however, the accretion rate is higher, and an accretion column is believed to form. Plasma is decelerated by radiation pressure in the column, and a radiative shock forms above the neutron star surface (Davidson 1973; Basko \& Sunyaev 1976). Above the radiative shock, plasma is in free fall, while below the discontinuity, there is a region of nearly stagnant plasma from which photons escape from the sides of the column in a fan beam.

In addition, a luminous halo might form around the accretion column (Davidson \& Ostriker 1973; Lyubarskii \& Syunyaev 1988) and radiation from the polar cap and the halo can also be scattered in the upper accretion stream (Soffel et al. 1985; Brainerd \& Meszaros 1991). As the accretion stream is delimited by magnetic field lines of the neutron star, it opens up wide far from the neutron star. Therefore, the emission from the upper part of the accretion stream can dominate the observed flux and can also screen the polar caps and the halo. Due to relativistic light deflection, emission from one pole can be deflected to the antipodal direction (Pechenick et al. 1983; Riffert \& Meszaros 1988; Leahy \& Li 1995; Kraus 2001). All these effects can modify the local beam pattern and thus have an effect on the pulse profile of the X-ray pulsar. Wang \& Welter (1981) have modeled the pulse profiles of X-ray pulsars by assuming hot spots and fan beams, although EXO $2030+375$ is not included in their list of selected X-ray pulsars. They show that, in general, sharp minima can be seen in the pulse profiles at the moment when the fan beam rotates behind the horizon of the neutron star and when it reappears on the side of the neutron star facing the observer. This is exactly what seems to be happening in EXO 2030+375 at phases 0.1 and 0.6 as the decomposition method has shown. 

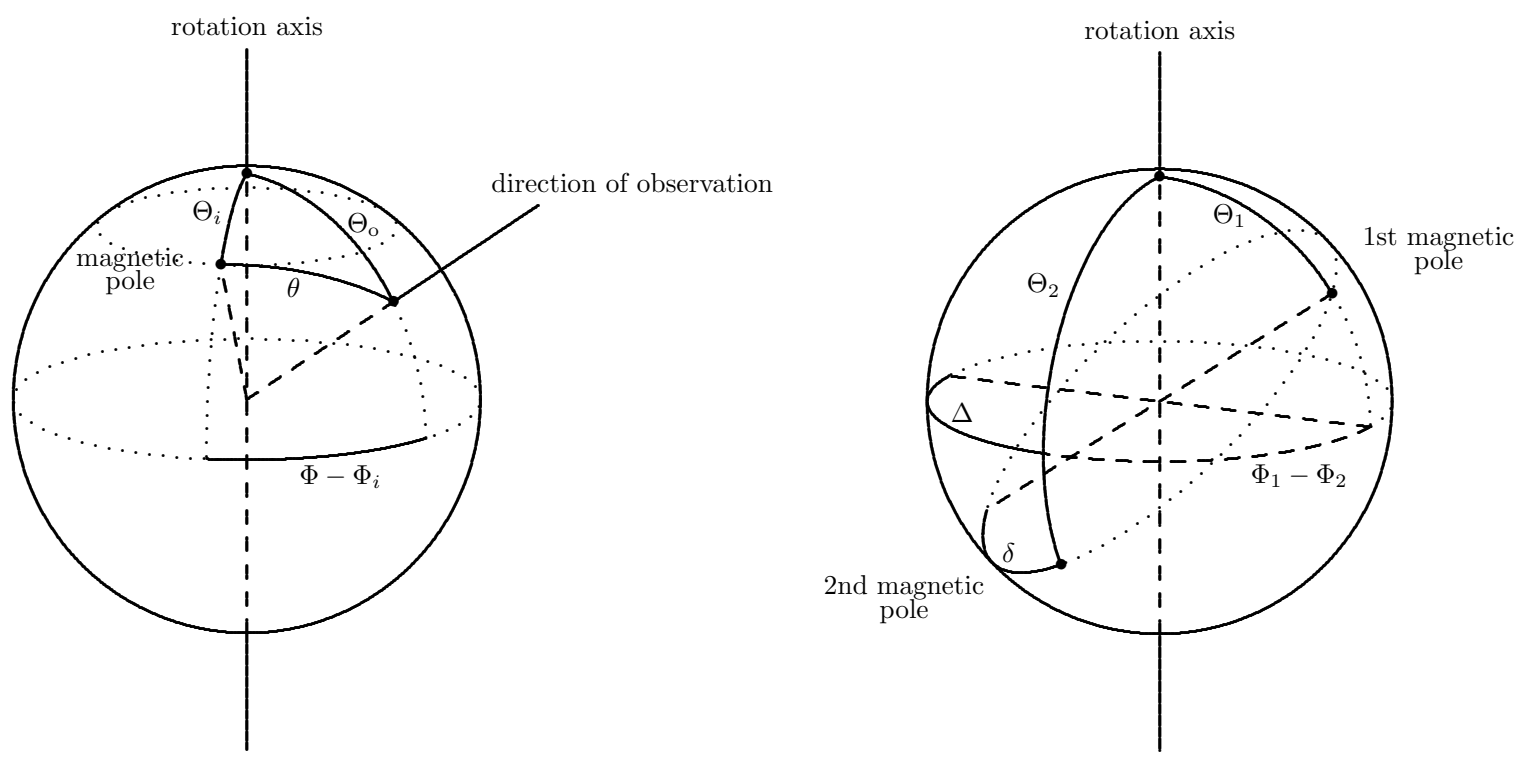

Fig. A.1. Intrinsic geometry of the neutron star. Figures taken from Kraus et al. (1995).

Kraus et al. $(2003,2010)$ have modeled beam patterns and pulse profiles for medium-luminosity X-ray pulsars assuming an accretion column with energy-dependent local beaming of radiation, a luminous halo formed by illumination of the neutron star surface, and magnetic scattering in the upper accretion stream. They show that the emission from the upper accretion stream can dominate the local beam pattern for $\theta>120^{\circ}$. Halo emission has a maximum at $\theta \approx 30^{\circ}-60^{\circ}$, with significant contribution to the beam pattern for photon energies below $\sim 5 \mathrm{keV}$. If one assumes isotropic emission from the accretion column below the discontinuity, the halo emission becomes comparable or negligible relative to the column emission at $\gtrsim 10 \mathrm{keV}$. However, if the radiation below the shock is beamed downwards, the halo remains more luminous than the accretion column and the upper stream up to $\sim 30 \mathrm{keV}$. The decomposition has shown that the beam patterns show dominant soft emission for $\theta<60^{\circ}$, which can be interpreted as halo emission, while the emission $\theta>150^{\circ}$ most likely arises from the upper accretion stream (see Fig. 3, lower panel).

\section{Summary}

We performed pulse-profile decomposition with data of EXO 2030+375 taken during the giant outburst of 2006 by RXTE and INTEGRAL. This is the fourth source after Cen X-3, Her X-1, and A $0535+26$ to which this method has been applied. Each of the asymmetric pulse profiles of EXO $2030+375$ at different luminosities in various energy bands are decomposed in two symmetric pulse profiles that account for emission from the two emission regions of the system.

We find that the magnetic field of the neutron star is moderately distorted. The observer sees a part of the emission from each of the two emission regions, but these parts do not overlap. We suggest that the main peak at phase 0.2 in the observed pulse profiles can be attributed to harder emission from the upper accretion stream of the second pole, which can be observed while the pole is on the other side of the neutron star because of relativistic light bending around the neutron star ("anti-pencil"). However, the main peak also has a considerable contribution from the halo emission of the first pole, which is closer to the line of sight than the second. Between phases $\sim 0.95$ and $\sim 0.1$, both poles are located on the rear side of the neutron star. The sharp minima seen at phases 0.0 and 0.6 are caused when the first pole is about to re- and disappear at the horizon of the neutron star, and its accretion column is seen from the side.

Our analysis has disentangled the emission components of the neutron star, which in total lead to the observed asymmetric, energy-, and luminosity-dependent pulse profiles of EXO 2030+375. It will allow us to perform detailed analyses of, e.g., the pulse phase-resolved spectra with reliable interpretation of the differences in the spectral parameters, hence shedding light on the physical processes in the system responsible for the observed emission.

Acknowledgements. This research is based on observations with INTEGRAL, an ESA project with instruments and science data centre funded by ESA member states (especially the PI countries: Denmark, France, Germany, Italy, Switzerland, Spain), Poland, and with the participation of Russia and the USA. This research has made use of data obtained from the High Energy Astrophysics Science Archive Research Center (HEASARC), provided by NASA's Goddard Space Flight Center. This work was supported by DFG grant SA 1777 1/1.

\section{Appendix A: The decomposition method}

The pulse-profile decomposition method has been developed and first presented by Kraus et al. (1995). Here we quickly summarize the method and the steps in the analysis.

\section{A.1. Decomposition into two single-pole pulse profiles}

Let $\theta$ be the angle between the direction of the line of sight and the axis through one magnetic pole. Figure A.1 shows the configuration of the neutron star, showing the positions of the magnetic poles with respect to the rotation axis. The polar angles of the two magnetic poles are called $\Theta_{1}$ and $\Theta_{2}$. As the neutron star rotates, $\theta$ changes with rotation angle $\Phi$. For the emission of each pole, there are two symmetry points at $\Phi_{i}$ and $\Phi_{i}+\pi(i=1,2)$. For each pole, the relation between $\theta, \Theta_{i}$, and $\Phi_{i}$ can be obtained using the spherical triangle in Fig. A.1 (left):

$\cos \theta=\cos \Theta_{0} \cos \Theta_{i}+\sin \Theta_{0} \sin \Theta_{i} \cos \left(\Phi-\Phi_{i}\right)$. 


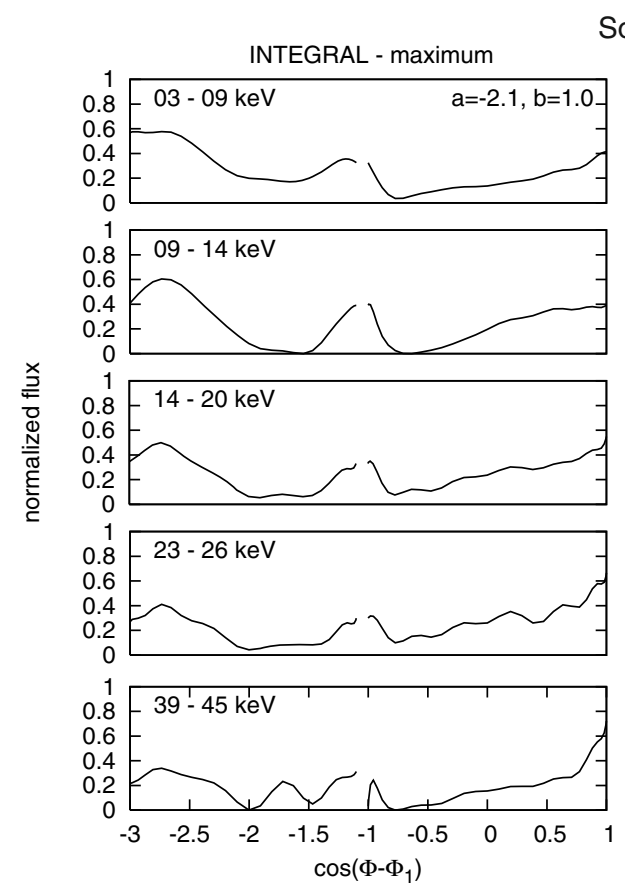

Solution 1
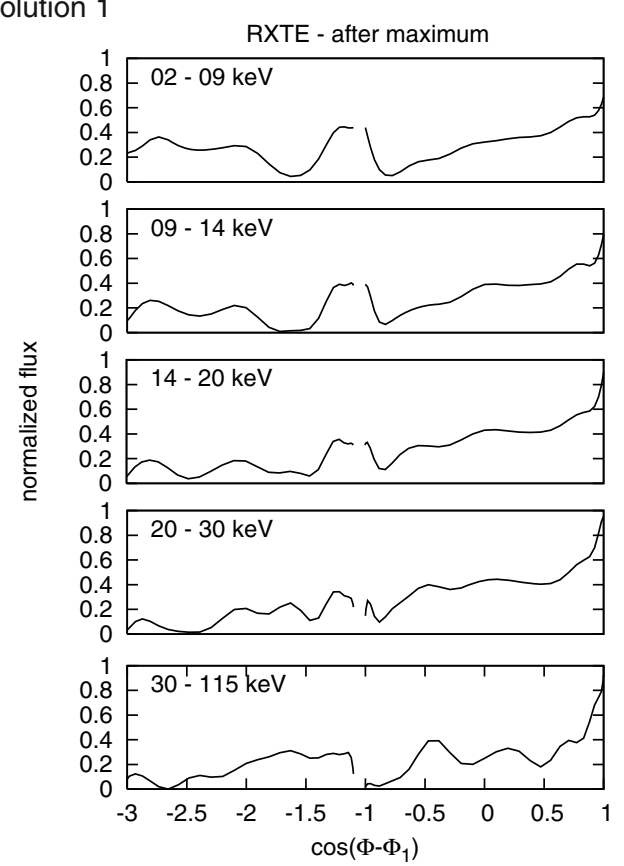

Solution 2

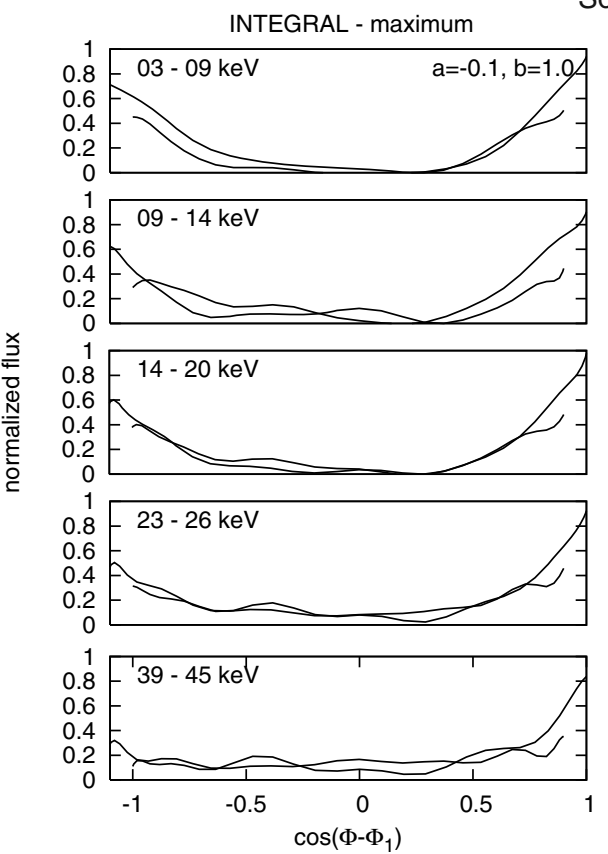

Fig. A.2. Beam patterns from the two emission regions seen by the observer plotted over $\cos \left(\Phi-\Phi_{1}\right)$. The left panel shows the beam patterns of solution 1 of the observation near maximum as shown in Fig. 2. The right panel shows a selection of additional data from the observation after maximum with similar energy bands. For solution 1 (upper panel) no overlap of the beam patterns for the two emission regions is found. For solution 2 (lower panel), the beam patterns seem to overlap over a wide range, but show small differences. For the parameters $a, b$, see Sect. 3.3.
We assume that there is an offset $\delta$ between the second magnetic pole and the antipodal position of the first magnetic pole, therefore, there will be a phase offset for the rotation angles $\Phi_{i}$ from a symmetric configuration which can be defined as

$\Delta=\pi-\left(\Phi_{1}-\Phi_{2}\right)$.

For an ideal dipole field $\Theta_{1}+\Theta_{2}=\pi, \Delta=0$, and $\delta=0$. With the polar angles $\Theta_{1}, \Theta_{2}$, and the offset $\Delta$ in the rotation angles, we have a complete set of parameters to describe the geometry of the neutron star.

To find the contributions from each magnetic pole, we perform Fourier analysis of the observed total pulse profiles. We model it as a sum of two symmetric functions $f_{1}$ and $f_{2}$ and search for the values for their symmetry points $\Phi_{1}$ and $\Phi_{2}$, respectively. In principle we are able to find a set of $f_{1}$ and $f_{2}$ for any chosen $\Phi_{1}$ and $\Phi_{2}$. However, since we deal with functions that describe astronomically observed emission, the following criteria need to be fulfilled:

1. Positive flux: The symmetric functions $f_{1}$ and $f_{2}$ must not have negative values because they model the flux of an astronomical object.

2. No ripples: The functions $f_{1}$ and $f_{2}$ should show no smallscale features that cancel out in the sum. As the two functions correspond to pulse profiles of single-poles that emit independently, they ought not to have features that match exactly. Also, the single-pole pulse profiles are supposedly not more complicated than the total pulse profile.

3. Same geometry: We have pulse profiles from one source in different energy bands and, ideally, from more than one 

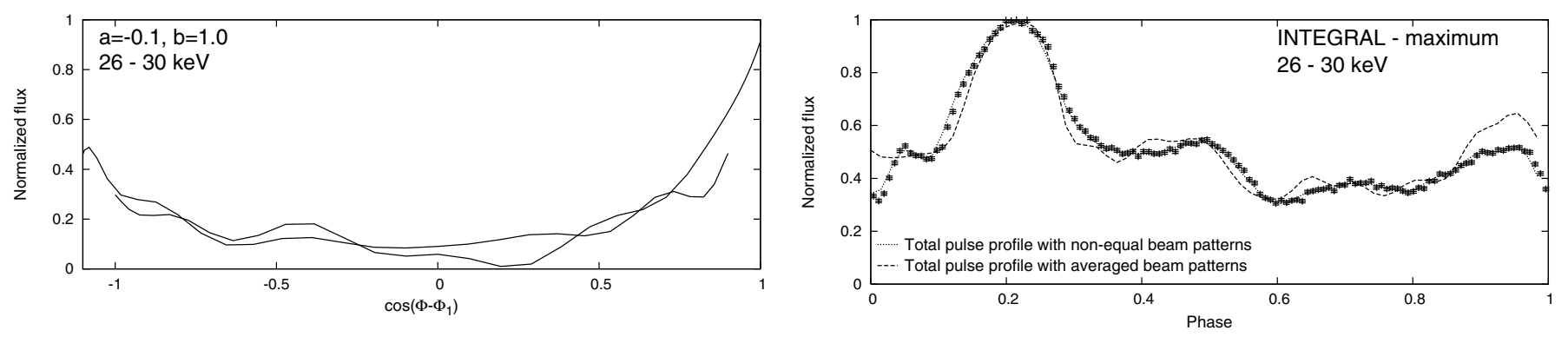

Fig. A.3. Left: beam patterns from the two emission regions plotted over $\cos \left(\Phi-\Phi_{1}\right)$ for the energy band $26-30 \mathrm{keV}$ of the INTEGRAL observation near maximum for solution 2. For this solution, the beam patterns seem to overlap over a wide range, but show small differences between each other. Right: observed total pulse profile (data points with errors) with total pulse profiles reconstructed from the not perfectly matching beam patterns of solution 2 for the two emission regions (dotted) and averaged beam patterns assuming equal local emission pattern for the two regions (dashed).

observation. The observed pulse profiles are energy and luminosity dependent in most cases. Since the emission arises from only one object, the symmetry points must be the same for the decompositions of all available data.

The actual search for reasonable decompositions is performed using the parameters $\Phi_{1}$ and $\Delta$. The second symmetry point $\Phi_{2}$ can then be obtained from Eq. (A.2). The decompositions of the pulse profiles of different energy bands and different observations will not yield identical values for $\Phi_{1}$ and $\Delta$. Therefore we have to define interesting regions in the parameter space of $\Phi_{1}$ and $\Delta$ and look at the single-pole pulse profile for similar values of $\Phi_{1}$ and $\Delta$ for each energy band and observation to decide if they can be declared as one consistent set of emission. This means that the single-pole pulse profiles have to have similar values of $\Phi_{1}$ and $\Delta$ and ought to be similar from one energy band to the other in one observation. Once one has found such a set of decomposition for all energy bands and observations for similar $\Phi_{1}$ and $\Delta$, one can compute the corresponding beam patterns for each pole.

\section{A.2. Reconstruction of the beam pattern}

Two beam patterns are obtained from single-pole pulse profiles as functions of the rotation angles $\Phi$ (for one single-pole pulse profile) and $\tilde{\Phi}$ (for the other single-pole pulse profile). During one revolution of the neutron star, the angle $\theta$ between the first magnetic pole and the line of sight changes with the phase. There may be an interval during one phase, i.e., a range of the angle $\theta$, in which the observer sees emission from both poles. The same is true for the second magnetic pole. The range covered by the angle $\theta$ is in general different for each pole. There may, however, be a certain subrange of values of $\theta$ that occur for each of the poles. Then, at some phase $\Phi$, the observer looks onto the first pole at angle $\theta$ and at some different phase $\tilde{\Phi}$, the observer looks onto the second pole at the same angle $\theta$. A simple example would be two antipodal poles that pass through the line of sight $(\theta=0)$ at $\Phi=0$ and at $\widetilde{\Phi}=0.5$, respectively. If, in addition, the two emission regions of the neutron star have the same beam pattern, the emission seen at $\theta$ from the first pole (phase $\Phi$ ) is the same as the emission seen at $\theta$ from the second pole (phase $\tilde{\Phi}$ ). This means that the visible beam patterns of the two poles must have identical parts at different pulse phases. If we find such parts of the beam patterns of the two single-pole pulse profiles we can overlay them and get a relation between $\cos \left(\Phi-\Phi_{1}\right)$ and $\cos \left(\tilde{\Phi}-\Phi_{2}\right)$. From this relation, we can derive the positions of the magnetic poles $\Theta_{1}$ and $\Theta_{2}$ as functions of the direction of the observation
$\Theta_{0}$. The angle $\Theta_{0}$ needs to be determined independently in other studies of the source.

\section{References}

Angelini, L., Stella, L., \& Parmar, A. N. 1989, ApJ, 346, 906 Basko, M. M., \& Sunyaev, R. A. 1976, MNRAS, 175, 395 Bildsten, L., Chakrabarty, D., Chiu, J., et al. 1997, ApJS, 113, 367

Blum, S., \& Kraus, U. 2000, ApJ, 529, 968

Brainerd, J. J., \& Meszaros, P. 1991, ApJ, 369, 179

Bulik, T., Riffert, H., Meszaros, P., et al. 1995, ApJ, 444, 405

Caballero, I., Kraus, U., Santangelo, A., Sasaki, M., \& Kretschmar, P. 2010, A\&A, submitted

Coe, M. J., Payne, B. J., Longmore, A., \& Hanson, C. G. 1988, MNRAS, 232, 865

Corbet, R. H. D., \& Levine, A. M. 2006, The Astronomer's Telegram, 843, 1

Davidson, K. 1973, Nature, 246, 1

Davidson, K., \& Ostriker, J. P. 1973, ApJ, 179, 585

Frontera, F., \& Dalfiume, D. 1989, in Two Topics in X-Ray Astronomy, Vol.

1, X Ray Binaries, Vol. 2, AGN and the X Ray Background, ed. J. Hunt, \& B. Battrick, ESA SP, 296, 57

Gehrels, N., Chincarini, G., Giommi, P., et al. 2004, ApJ, 611, 1005

Jahoda, K., Swank, J. H., Giles, A. B., et al. 1996, in SPIE Conf. Ser. 2808, ed. O. H. Siegmund, \& M. A. Gummin, 59

Janot-Pacheco, E., Motch, C., \& Pakull, M. W. 1988, A\&A, 202, 81

Klochkov, D., Horns, D., Santangelo, A., et al. 2007, A\&A, 464, L45

Klochkov, D., Santangelo, A., Staubert, R., \& Ferrigno, C. 2008, A\&A, 491, 833

Kraus, U. 2001, ApJ, 563, 289

Kraus, U., Nollert, H.-P., Ruder, H., \& Riffert, H. 1995, ApJ, 450, 763

Kraus, U., Blum, S., Schulte, J., Ruder, H., \& Meszaros, P. 1996, ApJ, 467, 794

Kraus, U., Zahn, C., Weth, C., \& Ruder, H. 2003, ApJ, 590, 424

Kraus, U., Zahn, C., \& Santangelo, A. 2010, A\&A, submitted

Krimm, H., Barthelmy, S., Gehrels, N., et al. 2006, The Astronomer's Telegram, 861,1

Leahy, D. A. 1991, MNRAS, 251, 203

Leahy, D. A., \& Li, L. 1995, MNRAS, 277, 1177

Lund, N., Budtz-Jørgensen, C., Westergaard, N. J., et al. 2003, A\&A, 411, L231

Lyubarskii, Y. E., \& Syunyaev, R. A. 1988, SvA Lett., 14, 390

McCollough, M. L., Turler, M., Willis, D., \& Shaw, S. E. 2006, The Astronomer's Telegram, 868, 1

Motch, C., \& Janot-Pacheco, E. 1987, A\&A, 182, L55

Parmar, A. N., White, N. E., \& Stella, L. 1989a, ApJ, 338, 373

Parmar, A. N., White, N. E., Stella, L., Izzo, C., \& Ferri, P. 1989b, ApJ, 338, 359

Pechenick, K. R., Ftaclas, C., \& Cohen, J. M. 1983, ApJ, 274, 846

Reynolds, A. P., Parmar, A. N., \& White, N. E. 1993, ApJ, 414, 302

Riffert, H., \& Meszaros, P. 1988, ApJ, 325, 207

Riffert, H., Nollert, H.-P., Kraus, U., \& Ruder, H. 1993, ApJ, 406, 185

Soffel, M., Herold, H., Ruder, H., \& Ventura, J. 1985, A\&A, 144, 485

Ubertini, P., Lebrun, F., Di Cocco, G., et al. 2003, A\&A, 411, L131

Wang, Y., \& Welter, G. L. 1981, A\&A, 102, 97

White, N. E., Swank, J. H., \& Holt, S. S. 1983, ApJ, 270, 711

Wilson, C. A., Finger, M. H., Coe, M. J., Laycock, S., \& Fabregat, J. 2002, ApJ,

570,287

Wilson, C. A., Fabregat, J., \& Coburn, W. 2005, ApJ, 620, L99

Wilson, C. A., Finger, M. H., \& Camero-Arranz, A. 2008, ApJ, 678, 1263

Winkler, C., Courvoisier, T. J.-L., Di Cocco, G., et al. 2003, A\&A, 411, L1 